\title{
Fasceítis necrotizante cervical: Consideraciones para el manejo precoz
}

\author{
Cervical necrotizing fascitis: considerations for early management \\ Tamara Barría $\mathrm{E}^{1}$, Agustín Abarca $\mathrm{S}^{2}$, Miguel Cancino $\mathrm{C}^{1}$, Cristóbal Chávez $\mathrm{S}^{3}$, José Parra $\mathrm{P}^{4}$, \\ Maritza Rahal E'.
}

\begin{abstract}
RESUMEN
La fasceítis necrotizante cervical (FNC) es una infección que afecta la fascia cervical y tejido subcutáneo, diseminándose rápidamente a través de los planos fasciales con una alta tasa de mortalidad. Si bien, las imágenes son una herramienta de apoyo fundamental para el diagnóstico, éste sigue siendo eminentemente clínico, presentando una rápida progresión de síntomas en pocas horas. El objetivo de esta presentación fue identificar factores descritos con peor pronóstico en el diagnóstico precoz de la FNC. Se realizó un estudio descriptivo de serie de casos de pacientes con diagnóstico de FNC en los últimos 10 años en el Servicio de Otorrinolaringología del Hospital Barros Luco Trudeau (HBLT). Se identificaron 5 pacientes, dentro de los cuales, los parámetros clínicos y de laboratorio a destacar fueron el dolor desproporcionado y rápido deterioro de exámenes de laboratorio. En los casos que había signos sugerentes de FNC en la tomografía computarizada, se favoreció el manejo quirúrgico agresivo, mientras que, en quienes no había imágenes sugerentes de FNC, se postergó el diagnóstico y su manejo precoz, provocando un desenlace fatal. La FNC constituye un cuadro grave en el que la sospecha clínica y rapidez de inicio del tratamiento resultan fundamental en el pronóstico.
\end{abstract}

Palabras clave: Fasceítis necrotizante, parámetros de laboratorio.

\begin{abstract}
Cervical necrotizing fasciitis (CNF) is an infection that affects the cervical fascia and subcutaneous tissue. It is characterized by a rapid dissemination trough the fascial planes, with a high rate of mortality. Even tough imaging results a fundamental diagnostic tool, it is still made by clinic signs with rapid progression of symptoms in few hours. Here, we identify worse prognostic factors in the precocious diagnosis of CNF, from five cases presented in our center. A descriptive case-series study was performed in patients with CNF in the last ten years in the Otolaryngology Department of Barros Luco Trudeau
\end{abstract}

\footnotetext{
Otorrinolaringólogo, Hospital del Salvador, Santiago, Chile.

Otorrinolaringólogo, Hospital Barros Luco Trudeau, Santiago, Chile.

3 Otorrinolaringología, Hospital Clínico Universidad de Chile, Santiago, Chile.

4 Otorrinolaringología, Hospital San Juan de Dios, Santiago, Chile.
}

Recibido el 22 de noviembre, 2017. Aceptado el 11 de enero, 2018. 
Hospital. Five patients were identified, between the clinical and laboratory parameters. A disproportional pain and rapid deterioration of the laboratory exams were the most highlighted features. In the cases with suggestive signs of CFN in the CT scan, allowed an aggressive surgical management, while in those that had no suggestive images the diagnosis was delayed and therefore their management ended in a fatal outcome. CFN constitutes a severe picture in wich clinical suspicion and a prompt initiation of treatment are fundamental in its prognosis.

Key words: Necrotizing fasciitis, score, laboratory parameters.

\section{INTRODUCCIÓN}

La fasceítis necrotizante cervical (FNC) corresponde a una infección fulminante que afecta a los tejidos blandos y conectivos, que se propaga a través del tejido celular subcutáneo y fascias del cuello, generando trombosis tanto arteriales como venosas, además de micro y macroembolías sépticas, lo que llevará a necrosis, sobreinfección y un gran compromiso del estado general ${ }^{1,2}$. Se caracteriza por una marcada toxicidad sistémica y un alto porcentaje de complicaciones que explican su elevada mortalidad, descrita según diferentes series publicadas entre $20 \%$ a $73 \% \%^{2,3}$. Corresponde a una entidad poco frecuente, descrita en mayor proporción en otros sitios anatómicos como extremidades, región perineal y abdomen, de los cuales sólo $5 \%$ se limitan a cabeza y cuello, con una incidencia de 3,5 a 4 casos por 100.000 habitantes por año, presentándose principalmente en población adulta ${ }^{4-7}$. El foco inicial de infección es variable, sin embargo se describe que fundamentalmente es de origen odontogénico o faríngeo (Figura 1), este último relacionado a patología amigdalina ${ }^{1}$.

Su microbiología exacta también es variable, pero mayormente corresponde a cultivos polimicrobianos, aunque un porcentaje no menor corresponden a infecciones por Staphylococcus aureus y/o Streptococcus pyogenes. Cabe destacar que en aproximadamente $9 \%$ de los casos el cultivo puede resultar negativo. En estos casos los hallazgos intraoperatorios y biopsia resultan fundamentales ${ }^{1,2}$. El retraso tanto en la sospecha diagnóstica como en el inicio del tratamiento conllevan a una serie de complicaciones, entre las que destacan sepsis, mediastinitis, erosión de la arteria carótida, tromboflebitis yugular, neumonía por aspiración, entre otras, siendo más frecuentes la sepsis y mediastinitis, lo cual explica la variabilidad y alto porcentaje de mortalidad de esta patología $a^{7-10}$.

Corresponde a una patología rápidamente progresiva, pudiendo pasar desde los primeros síntomas hasta la instalación de un cuadro grave en menos de $48 \mathrm{~h}^{11}$. La sospecha diagnóstica se basa en hallazgos clínicos, tanto anamnésticos como

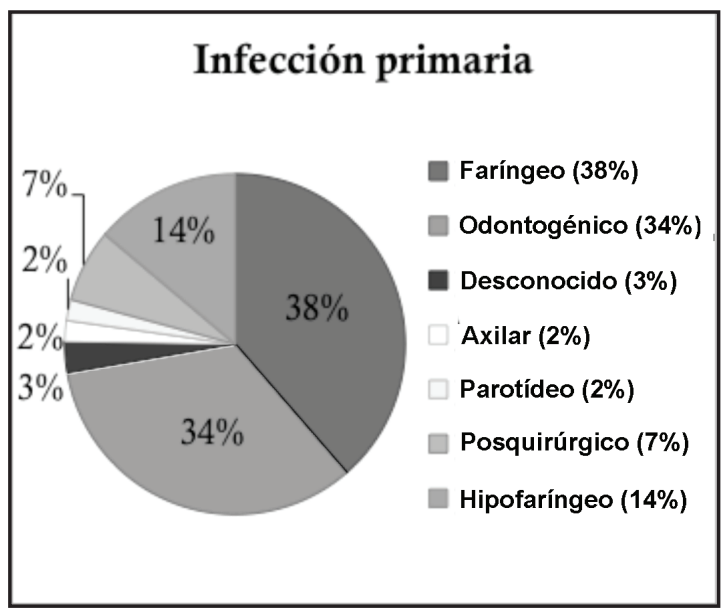

Figura 1. Infección primaria en fasceítis necrotizante cervical. 
de examen físico, en la presencia de factores de riesgos y apoyados en elementos imagenológicos como de laboratorio. Mientras que el diagnóstico definitivo estará dado por los hallazgos intraoperatorios, biopsia y cultivo ${ }^{7-11}$. Los hallazgos clínicos más frecuentemente descritos corresponden a aumento de volumen, edema y eritema, asociado a un dolor desproporcionado y compromiso sistémico. En la medida que el cuadro progresa se agregarán síntomas como hipostesia, crépitos y necrosis visible ${ }^{11-14}$. En cuanto a los exámenes de apoyo, existen distintas escalas que buscan en base a alteraciones en los exámenes de laboratorio, establecer una mayor o menor probabilidad de presentar una fasceítis necrotizante. El más conocido es el escore de LRINEC (The laboratory risk indicator for cervical necrotizing fasciitis) que mide parámetros como hemoglobina, recuento de glóbulos blancos, plaquetas, PCR, natremia, creatinina y glicemia. Un puntaje mayor 0 igual a 6 determina que el diagnóstico es probable. Clasificando la sospecha diagnóstica según puntaje en "poco probable" si es menor a 6 puntos, "sospecha de FNC" si es mayor 0 igual a 6 y como "FNC altamente probable" si presenta un puntaje mayor 0 igual a 8 . Se ha descrito que este escore tendría un alto valor predictivo negativo (99\%) y bajo valor predictivo positivo $(29 \%)$, con una sensibilidad de $77 \%$ a $94 \%$ y especificidad de $94 \%$ (Tabla 1$)^{11}$.

A su vez, la tomografía computarizada (TC) de cuello con contraste puede mostrar hallazgos categóricos como enfisema subcutáneo, pérdida de planos fasciales, otros menos categóricos como miositis o realce graso, o incluso ser un examen normal ${ }^{11}$. Basándonos en esta información, en este trabajo evaluamos una serie de casos de FNC, con el objetivo de identificar los factores descritos con peor pronóstico para el diagnóstico precoz de esta patología.

\section{CASOS CLÍNICOS}

Se realizó un estudio descriptivo de serie de casos, en el que se revisaron fichas clínicas de pacientes con diagnóstico de fasceítis necrotizante cervical manejados por el equipo de otorrinolaringología del Hospital Barros Luco Trudeau entre los años 2006 a 2016. Se registraron distintas variables epidemiológicas, clínicas y temporales asociadas, como la edad, sexo, comorbilidades, foco infeccioso de origen, síntoma principal de consulta, tiempo transcurrido hasta el diagnóstico, puntaje escore LRINEC, hallazgos en tomografía computarizada de cuello con contraste (catalogándose como signos sugerentes la presencia de aire, colecciones 0 pérdida de los planos fasciales en ésta) y la mortalidad.

Se pesquisaron 5 casos diagnosticados como fasceítis necrotizante cervical en base a hallazgos intraoperatorios como a los resultados de cultivo y biopsia. De los 5 casos, tres correspondieron a pacientes de sexo femenino, con un promedio de edad fue de 41,6 años y un rango etario de 30 a 60 años. El foco infeccioso inicial más frecuente fue amigdalino (3 casos), y la sintomatología más frecuente fue aumento de volumen cervical (4 casos), asociado a eritema y dolor desproporcionado, presentando a su vez, en la mayoría de los casos (3 casos) algún signo de compromiso sistémico. Mientras que sólo en un caso se encontró crépitos al examen físico (Tabla 2 y Figura 2).

En esta serie de casos, se observó una discordancia entre el valor del escore de LRINEC y

Tabla 1. Puntaje LRINEC. Adaptado de Sandner et al. J Oral Maxillofac Surg 2015

\begin{tabular}{lc} 
Variable & Escore LRINEC \\
PCR (mg/dl) & \\
$<150$ & 0 \\
$\leq 150$ & 4 \\
Recuento de blancos & \\
$<15$ & 0 \\
$15-25$ & 1 \\
$>25$ & 2 \\
Hemoglobina (g/dl) & \\
13,5 & 0 \\
$11-13,5$ & 1 \\
$<11$ & 2 \\
Sodio (umol/L) & \\
$\geq 135$ & 0 \\
$<135$ & 2 \\
Creatinina (umol/L) & \\
$\leq 141$ & \\
$>141$ & 2 \\
Glucosa & \\
$\leq 100$ & 1 \\
$>100$ & \\
\hline
\end{tabular}


Tabla 2. Hallazgos clínicos

\begin{tabular}{|c|c|c|c|c|c|c|c|c|}
\hline $\begin{array}{l}N^{0} \text { caso/ Sexo } \\
\text { (años) }\end{array}$ & Edad & Comorbilidad & $\begin{array}{c}\text { Foco infeccioso } \\
\text { de origen }\end{array}$ & $\begin{array}{l}\text { Síntoma o signos } \\
\text { principales }\end{array}$ & $\begin{array}{l}\text { Escore } \\
\text { LRINEC }\end{array}$ & $\begin{array}{l}\text { Presencia de } \\
\text { TC cervical } \\
\text { sugerente/ } \\
\text { mediastinitis }\end{array}$ & $\begin{array}{c}\text { Tiempo } \\
\text { desde } \\
\text { ingreso a } \\
\text { diagnóstico }\end{array}$ & Defunción \\
\hline 1/ Femenino & 53 & $\begin{array}{c}\text { Diabetes } \\
\text { Mellitus } 2\end{array}$ & Dental & $\begin{array}{l}\text { Aumento } \\
\text { de volumen }\end{array}$ & 5 & $+1-$ & 12 horas & No \\
\hline 2/ Femenino & 31 & Poliadicción & $\begin{array}{l}\text { Adenitis } \\
\text { cervical }\end{array}$ & $\begin{array}{l}\text { Dolor intenso } \\
\text { desproporcionado, } \\
\text { crépitos }\end{array}$ & 8 & $+1+$ & 4 horas & No \\
\hline 3/ Masculino & 60 & Obesidad & Amigdalino & $\begin{array}{l}\text { Aumento de } \\
\text { volumen y falla } \\
\text { orgánica múltiple }\end{array}$ & 8 & $-1-$ & 6 días & Sí \\
\hline 4/ Masculino & 34 & - & Amigdalino & $\begin{array}{l}\text { Aumento de volumen } \\
\text { y compromiso del } \\
\text { estado general }\end{array}$ & 4 & $+1+$ & 12 horas & Sí \\
\hline 5/ Femenino & 30 & - & Amigdalino & $\begin{array}{l}\text { Aumento de volumen, } \\
\text { dolor intenso } \\
\text { desproporcionado y } \\
\text { compromiso del } \\
\text { estado general }\end{array}$ & 7 & $+1-$ & 10 horas & No \\
\hline
\end{tabular}
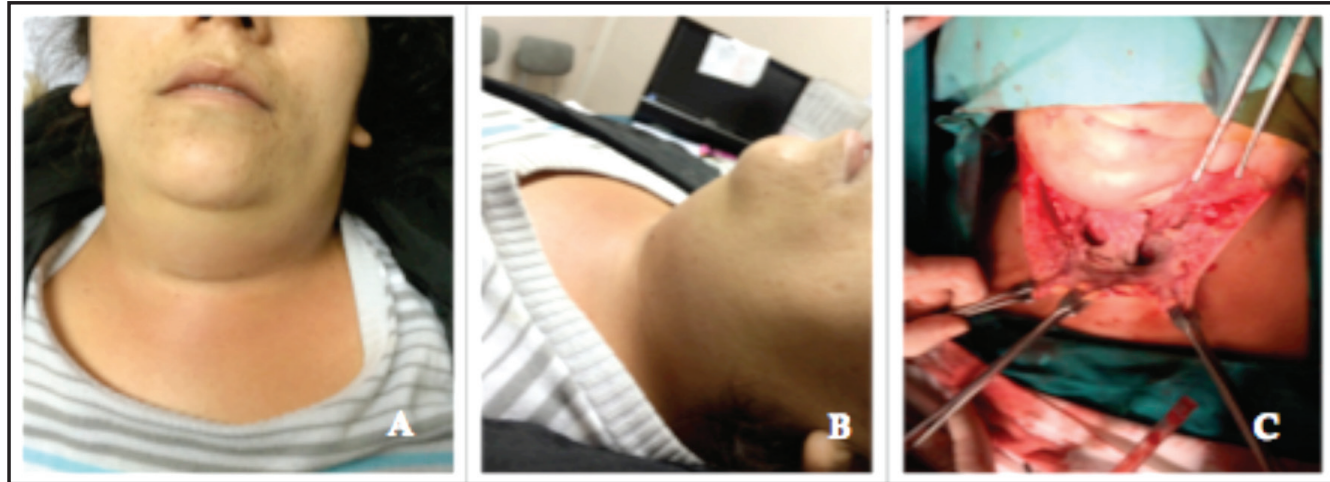

Figura 2. Caso clínico n². (A) y (B) Aumento de volumen cervical y eritema local. (C) Hallazgos en intraoperatorio con tejido necrótico y pérdida de planos fasciales.

los hallazgos del TC cervical con contraste. Dos casos, presentaron un escore LRINEC bajo (menor a 6 puntos, que se clasificaría como baja probabilidad de FNC) pese a tener un contexto clínico y TC cervical con contraste sugerentes, incluso uno de los casos presentó una complicación grave como mediastinitis. Así mismo, en un caso el escore sugirió una alta probabilidad de fasceítis, pese a no tener imagenología sugerente. Mientras que sólo 2 casos fueron concordantes clínica e imagenológicamente, clasificándose por puntaje LRINEC en pacientes con "sospecha de FNC" y "altamente probable de FNC", teniendo imágenes compatibles con FNC sin mediastinitis y FNC con mediastinitis, respectivamente (Tabla 2).

En cuanto al tiempo de demora desde el ingreso al hospital hasta la sospecha diagnóstica de FNC, 4 pacientes fueron diagnosticados antes 
de las primeras 15 horas desde el ingreso, destacando la presencia de imagenología sugerente de FNC en todos estos casos. Mientras que sólo un caso tuvo un diagnóstico tardío, coincidiendo con que fue el único caso que no presentó signos sugerentes de FNC y un escore LRINEC altamente probable (Tabla 2).

Todos los pacientes requirieron más de una intervención quirúrgica, consistente en aseo profuso y desbridamiento agresivo de tejido necrótico, con un promedio de 3,2 intervenciones. Todos fueron manejados en unidad de cuidados intensivos, con un promedio de estadía de 23,8 días. Finalmente, en cuanto al desenlace, 2 de los 5 pacientes fallecieron $(40 \%)$, destacándose como factores relevantes, la presencia de mediastinitis concomitante en un caso y el diagnóstico (y por ende, manejo) tardío en el otro.

\section{DISCUSIÓN}

La FNC corresponde a un cuadro dramático, de rápida progresión y elevada mortalidad, según lo evaluado en este trabajo y lo descrito en la literatura. El diagnóstico definitivo se basa en hallazgos intraoperatorios; como un tejido desvitalizado, con una coloración rojo-grisácea pálida, poco sangrante $\mathrm{y}$ con venas superficiales con signos de trombosis; biopsia y cultivo, el cual puede ser negativo incluso en hasta $9 \%$ de los casos. Sin embargo, la sospecha clínica precoz resulta fundamental dado lo variable de los hallazgos imagenológicos (donde la TC presenta una sensibilidad intermedia, pero alta

\section{BIBLIOGRAFÍA}

1. Elander J, Nekludov M, Larsson A, Nordlander B, Eksborg S, Hydman J. Cervical Necrotizing Fasciitis: descriptive, retrospective analysis of 59 cases treated at a single center. Eur Arch Otorhinolaryngol 2016; 273: 4461-67. doi: 10.1007/s00405-016-4126-y.

2. Cruz Toro P, Callejo Castillo À, Tornero Saltó J, González Compta X, Farré A, Maños M. Cervical necrotizing fasciitis: report of 6 cases and especificidad) y la inespecificidad de los exámenes de laboratorio. En nuestra serie destaca que en 4 de Ios casos se encontraron signos sugerentes de FNC en la TC, favoreciendo el manejo quirúrgico agresivo, mientras que en el paciente que no presentaba imágenes sugerentes se postergó el diagnóstico y por ende su manejo precoz provocando un desenlace fatal, por este motivo, creemos que es importante enfatizar que ante un compromiso clínico desproporcionado para los hallazgos físicos e imagenológicos, debemos sospechar igual este diagnóstico, para evitar desenlaces lamentables.

La sospecha es clínica, en contexto de un cuadro con sintomatología sugerente (como aumento de volumen, eritema y dolor intenso, con una clínica no concordante, ya sea por la rápida progresión de los síntomas, por un compromiso sistémico importante) o por lo intenso de la sintomatología en relación a los hallazgos del examen físico. El laboratorio e imágenes apoyan y refuerzan la sospecha, pero la indicación de exploración quirúrgica sigue siendo clínica como se pudo reflejar en nuestra casuística.

\section{CONCLUSIÓN}

El diagnóstico de la FNC es un desafío clínico, no existen exámenes de certeza, por lo que la clínica sigue siendo lo más importante para su sospecha diagnóstica. El manejo precoz y agresivo determina la supervivencia en este cuadro, por lo que ante la sospecha se debe actuar y explorar quirúrgicamente. review of literature. Eur Ann Otorhinolaryngol Head Neck Dis 2014; 131: 357-9. doi: 10.1016/j. anorl.2013.08.006.

3. Lin C, Yeh FL, Lin JT, Ma H, Hwang CH, Shen BH, ET AL. Necrotizing fasciitis of the head and neck: an analysis of 47 cases. Plast Reconstr Surg 2001; 107: 1684-93.

4. Endorf FW, Cancio LC, Kelin MB. Necrotizing soft tissue infections: Clinical guidelines. $J$ Burn Care Res 2009; 30: 769-75. doi: 10.1097/ BCR.0b013e3181b48321. 
5. Simonsen E, van Orman eR, Hatch BE, et al. Cellulitis incidence in a defined population. Epidemiol Infect 134: 293, 20066.

6. Shaikh N, Ummunissa F, Hanssen Y, Al Makki H, SHOKR HM. Hospital epidemiology of emergent cervical necrotizing fasciitis. J Emerg Trauma Shock 2010; 3: 123-5. doi: 10.4103/09742700.62108.

7. Fenton CC, Kertesz T, Baker G, Sandor GK. Necrotizing fasciitis of the face: a rare but dangerous complication of dental infection. $J$ Can Dent Assoc 2004; 70: 611-5.

8. Flanagan CE, Daramola 00, Maisel RH, Adkinson C, Odland RM (2009). Surgical debridement and adjunctive hyperbaric oxygen in cervical necrotizing fasciitis. Otolaryngol-Head Neck Surg: Off J Am Acad Otolaryngol-Head Neck Surg 140(5): 730-4.

9. Sarna T, Sengupta T, Miloro M, Kolokythas A. Cervical necrotizing fasciitis with descending mediastinitis: literature review and case report.
J Oral Maxillofac Surg 2012; 70: 1342-50.

10. Lanisnik $B$, CIzMarevic $B$. Necrotizing fasciitis of the head and neck: 34 cases of a single institution experience. Eur Arch Otorhinolaryngol 2010; 267: 415-21.

11. Sandner A, Moritz S, Unverzagt S, Plontke SK, Metz D. Cervical Necrotizing Fasciitis-The Value of the Laboratory Risk Indicator for Necrotizing Fasciitis Score as an Indicative Parameter. J Oral Maxillofac Surg 2015; 73: 2319-33.

12. Malik V, Gadepalli C, Agrawal S, Inkster C, Lobo C. An algorithm for early diagnosis of cervicofacial necrotising fasciitis. Eur Arch Otorhinolaryngol 2010; 267: 1169-77.

13. Lee JW, Immerman SB, Morris LG. Techniques for early diagnosis and management of cervicofacial necrotising fasciitis. J Laryngol Otol 2010; 124 : 759-64. doi: 10.1017/S0022215110000514.

14. Daszenies, M Rahal, R Arregui. Fascitis Necrotizante de cuello. Rev Otorrinolaringol Cir Cabeza Cuello 2004; 64: 247-51.

Dirección: Tamara Barría Espinoza 